\title{
COORDENADORES DE SAÚDE BUCAL: PERCEPÇÃO SOBRE GESTÃO E COMPETÊNCIAS NO SISTEMA ÚNICO DE SAÚDE
}

\author{
Oral health coordinators: perception on management and skills in the single health \\ system
}

\author{
Coordinadores de salud bucal: percepción de gestión y competencias del sistema \\ único de salud
}

\author{
Davi Oliveira Bizerril iD \\ Universidade de Fortaleza - UNIFOR - Fortaleza (CE) - Brasil
}

Francisco Cristóvão Mota Lima Júnior (iD

Secretaria Municipal de Saúde de Pacatuba - SMS/Pacatuba - Pacatuba (CE) - Brasil

Margarida Maria Saraiva iD

Secretaria Municipal de Saúde de Fortaleza - SMS/Fortaleza - Fortaleza (CE) - Brasil

Dulce Maria de Lucena Aguiar iD

Universidade de Fortaleza - UNIFOR - Fortaleza (CE) - Brasil

\section{RESUMO}

Objetivo: Analisar as percepções de coordenadores de saúde bucal sobre gestão e competências no Sistema Único de Saúde. Métodos: Estudo qualitativo realizado em seis Secretarias Executivas Regionais de Fortaleza, Ceará, Brasil. A coleta de dados ocorreu em março de 2019, através de entrevistas semiestruturadas com seis cirurgiões-dentistas que atuam à frente das coordenadorias de saúde bucal dos referidos órgãos. Analisaram-se as entrevistas por meio da análise de conteúdo, emergindo as seguintes categorias: competências de um coordenador de saúde bucal; curso de formação/capacitação para o cargo de gestor; contribuição da formação acadêmica ao cargo de gestor; desafios no processo de trabalho; satisfação por estar no cargo de gestor. Resultados: As principais atividades desenvolvidas pelos coordenadores de saúde bucal são: coordenação de recursos humanos; gerenciamento de material, insumos e equipamentos odontológicos; consolidação de procedimentos; planejamento de metas assistenciais e de promoção e prevenção em saúde bucal; produção e monitoramento de indicadores; e elaboração de relatórios técnicos. Segundo os participantes, o cargo de gestor requer capacitação na área de gestão em saúde pública, envolvimento e comprometimento na execução dos processos, além de ser essencial para a gestão de saúde bucal. Conclusão: Os coordenadores de saúde bucal consideram importante a gestão de saúde pública, apesar de obstáculos no cotidiano de trabalho, como quantidade insuficiente de insumos e subfinanciamento do setor, e necessidade de qualificação para assumir o cargo de gestor em saúde bucal para potencializar a atenção primária em saúde, promovendo ações de promoção, prevenção e recuperação da saúde do usuário.

Descritores: Gestão em Saúde; Saúde Coletiva; Saúde Bucal; Sistema Único de Saúde.

\section{ABSTRACT}

Objective: To analyze the perceptions of oral health coordinators about management and competencies in the Unified Health System. Methods: Qualitative study conducted in six Regional Executive Offices of Fortaleza, Ceará, Brazil. Data collection took place in March 2019, through semi-structured interviews with six dental surgeons who are in charge of the oral health coordinations of the referred organs. The interviews were analyzed through content analysis, emerging the following categories: competencies of an oral health coordinator; training course for the position of the manager; the contribution of academic formation to the position of the manager; challenges in the work process; satisfaction to be in the position of manager. Results: The main activities developed by oral health coordinators are: human resources coordination; management of dental materials, supplies and equipment; consolidation of procedures; planning of care goals and promotion and prevention in oral health; production and monitoring of indicators; and preparation of technical reports. According to the participants, the position of manager requires training in public health management, involvement, and commitment in the execution of processes, and is essential for oral

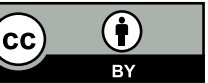


health management. Conclusion: Oral health coordinators consider public health management important, despite obstacles in daily work, such as insufficient inputs and underfunding of the sector, and the need for qualification to assume the position of oral health manager to enhance attention to health promotion, promoting actions for the promotion, prevention, and recovery of the user's health.

Descriptors: Health Management; Public Health; Oral Health; Unified Health System.

\section{RESUMEN}

Objetivo: Analizar las percepciones de coordinadores de salud bucal sobre gestión y competencias del Sistema Único de Salud. Métodos: Estudio cualitativo realizado en seis Secretarias Ejecutivas Regionales de Fortaleza, Ceará, Brasil. La recogida de datos se dio en marzo de 2019 a través de entrevistas semiestructuradas con seis cirujanos-odontólogos que actúan en las coordinaciones de salud bucal de los referidos órganos. Se analizaron las entrevistas a través del análisis de contenido de las cuales emergieron las siguientes categorías: competencias del coordinador de salud bucal; curso de formación/capacitación para el cargo de gestión; contribución de la formación académica para el cargo de gestión; los desafíos para el proceso de trabajo; satisfacción del cargo de gestión. Resultados: Las principales actividades desarrolladas por los coordinadores de salud bucal son: la coordinación de recursos humanos; el gerenciamiento de material, insumos y equipos odontológicos; la consolidación de procedimientos; el planeamiento de metas de asistencia y para la promoción y prevención de la salud bucal; la producción y el monitoreo de los indicadores; y la elaboración de informes técnicos. Según los participantes, el cargo de gestión requiere la capacitación para el área de la salud pública, el envolvimiento y el compromiso para la ejecución de los procesos además de ser esencial para la gestión de la salud bucal. Conclusión: Los coordinadores de salud bucal consideran importante la gestión de salud pública pese los obstáculos del cotidiano de trabajo como la cantidad insuficiente de insumos y la baja financiación del sector, y la necesidad de cualificación para asumir el cargo de gestión de salud bucal para potencializar la atención primaria de salud promoviendo acciones de promoción, prevención y recuperación de la salud del usuario.

Descriptores: Gestión en Salud; Salud Pública; Salud Bucal; Sistema Único de Salud.

\section{INTRODUÇÃO}

A gestão pública em saúde é uma área primordial, permeada de influência política, a qual oferece subsídios para criar e aplicar políticas, gerenciar e avaliar processos e programas, garantindo segurança para o paciente e conforto às equipes de saúde ${ }^{(1)}$.

No Sistema Único de Saúde (SUS), a gestão é um pilar básico para que o sistema funcione de uma forma equânime e universal. A saúde bucal é parte integrante desse sistema e apresenta uma organização estrutural de gestão que necessita ser acompanhada e melhorada. A Atenção Primária em Saúde (APS) apresenta-se como coordenadora e ordenadora das redes de atenção à saúde, com o intuito de dar cobertura a toda a população(2). Nela encontra-se a saúde bucal como uma área transversal a todos os programas e estratégias da APS.

A Política Nacional de Saúde Bucal (PNSB), conhecida como "Brasil Sorridente", lançada em 2004, com progressivas articulações transversais em ações dentro do Ministério da Saúde, junto a outros ministérios, tem como uma de suas proposições a reorientação da assistência em saúde bucal( ${ }^{(3)}$. Um dos eixos da PNSB é o de proteção e promoção da saúde, que inclui ações de cunho preventivo, prioritariamente instituídas para evitar ou minimizar doenças bucais (implantação de sistemas de fluoretação de águas nos municípios brasileiros e educação em saúde direcionada à assepsia oral) ${ }^{(4)}$.

Então, para o desenvolvimento de atividades de gestão eficazes referentes à atenção em saúde bucal, uma série de estratégias consideradas fundamentais, dentre elas os recursos humanos, as técnicas e as teorias de programação, são de grande relevância para a organização dos serviços, além dos processos, o trabalho assistencial e gerencial/gestor amadurecidos ${ }^{(5)}$.

A gestão de sistemas de serviços e de programas de saúde bucal é uma questão primordial. Sua importância pode ser identificada pelo desenvolvimento dos sistemas de proteção social com base em um modelo institucional redistributivo, o qual pressupõe urna ação consistente do Estado, que garanta bens e serviços a todos os cidadãos ${ }^{(6)}$.

A saúde bucal no SUS é considerada um processo social em construção contínua, com várias conquistas, em que as discussões a respeito do seu modelo de atenção precisam ser debatidas, assim como os paradigmas existentes no processo saúde-doença bucal e a atuação dos diferentes profissionais envolvidos ${ }^{(7)}$.

A Odontologia apresenta um histórico de práticas por meio de intervenção individual e clínica( ${ }^{(8)}$. Esse quadro tem se modificado com o aparecimento de cirurgiões-dentistas com perfil preventivista e gerencial. Essa prática 
de atenção à saúde bucal, basicamente focada no modelo clínico, que objetiva a solução do quadro de saúde do paciente, além do isolamento do cirurgião-dentista frente à função de ser o único responsável da área da saúde a cuidar do bem-estar bucal de um indivíduo, contribuem, de forma significativa, para as dificuldades encontradas na atuação dos cirurgiõesdentistas no SUS ${ }^{(5)}$.

Então, a participação de gestores na saúde bucal é recente, mas precisa ser amadurecida em prol de uma gestão compartilhada e interprofissional, além de oferecer uma prática de atenção à saúde bucal holística. Porém existem inúmeros obstáculos no processo de trabalho do gestor de saúde bucal, a exemplo da falta de recursos humanos e ingerências de recursos financeiros, demonstrando uma incompatibilidade entre seu processo de trabalho e as intervenções sociais desejadas ${ }^{(6)}$.

Diante da observação dos autores acerca das dificuldades enfrentadas no exercício da função de coordenador de saúde bucal, fez-se necessário o desenvolvimento desta pesquisa, com vistas a subsidiar o processo de trabalho de gestores em saúde bucal e futuras pesquisas sobre o tema.

As diretrizes da Política Nacional de Saúde Bucal( ${ }^{(4)}$ elencam ações desenvolvidas pelos coordenadores de saúde bucal, dentre elas: avaliação de indicadores, articulação entre os diferentes níveis de atenção e planejamento estratégico. Este estudo é importante, portanto, não só pela crescente presença do gestor de saúde bucal no Sistema Único de Saúde, como também pela orientação junto às secretarias estaduais e municipais sobre a importância do papel desse profissional nas redes de atenção à saúde. Assim, questiona-se: qual a percepção de coordenadores de saúde bucal sobre gestão e competências no Sistema Único de Saúde?

Diante desse contexto, objetivou-se analisar as percepções de coordenadores de saúde bucal sobre gestão e competências no Sistema Único de Saúde.

\section{MÉTODOS}

O estudo caracteriza-se como qualitativo. A pesquisa qualitativa trabalha com o universo de significados, motivos, aspirações, crenças, valores e atitudes, o que corresponde a um espaço mais profundo das relações, dos processos e dos fenômenos que não podem ser reduzidos à operacionalização de variáveis ${ }^{(9)}$.

Realizou-se o estudo em seis Secretarias Executivas Regionais (SER) em Fortaleza, Ceará, Brasil, divisão administrativa do município de Fortaleza, em que cada regional possui uma Coordenadoria de Educação, Meio Ambiente, Assistência Social e Infraestrutura, Saúde, dentre outras. No âmbito da Secretaria Municipal da Saúde (SMS), existe uma Coordenadoria de Saúde. Subordinada a esta, encontra-se a Coordenação Regional de Saúde Bucal, responsável por gerir a atenção em saúde bucal da população do município ${ }^{(10)}$.

Participaram do estudo seis cirurgiões-dentistas que atuam no cargo de gestão de coordenador de saúde bucal das seis SER do município de Fortaleza, Ceará, Brasil. De início, os profissionais em questão foram contatados através de ligações telefônicas e e-mails, nos quais se explicou a relevância, o propósito e a metodologia da pesquisa a ser realizada. Todos os participantes aceitaram participar do estudo. Utilizaram-se como critério de inclusão: ser coordenador de saúde bucal, independentemente do tempo no cargo, e estar disponível para submeter-se à entrevista no dia aprazado. Utilizaram-se como critérios de exclusão: não estar presente no dia da entrevista aprazada, sem oportunidade de outro dia para realização.

A coleta de dados ocorreu em março de 2019, por meio de entrevista semiestruturada, pois tem como característica questionamentos básicos que são apoiados em teorias e hipóteses relacionadas ao tema da pesquisa. Os questionamentos dariam frutos a novas hipóteses, surgidas a partir das respostas dos informantes. Caracterizase por um roteiro com perguntas abertas e fechadas ${ }^{(11)}$. O roteiro de entrevista tratou dos aspectos pessoais e profissionais dos entrevistados (idade, período de atuação, especialização, formação específica para atuação como gestor), além das seguintes questões norteadoras: quais as competências de um coordenador de saúde bucal? O curso de formação/capacitação do cargo contribuiu para assumir o cargo de gestor em saúde bucal? Qual a contribuição da formação acadêmica para o cargo? Quais os desafios no processo de trabalho? Qual a satisfação de estar no cargo como gestor?

O pesquisador responsável realizou a entrevista com o participante em um local reservado, nas dependências da sua respectiva SER. A entrevista ocorreu em clima de cordialidade entre ambos, com duração de 20 minutos, em média. Não houve critério de saturação da amostra, pois foi previamente estabelecida e contemplou os seis coordenadores de saúde bucal das SER, não havendo negação de nenhum coordenador em participar do estudo.

$\mathrm{Na}$ análise dos dados, avaliaram-se as descrições das entrevistas fornecidas pelos participantes segundo a análise de conteúdo ${ }^{(12)}$, que se apresenta como um conjunto de doze técnicas de análise das comunicações visando a obter, por procedimentos sistemáticos e objetivos de descrição do conteúdo das mensagens, indicadores 
quantitativos ou qualitativos que permitam a inferência de conhecimentos relativos às condições de produção e/ ou recepção de dados para discussão sob a luz da literatura pertinente. Na presente pesquisa, optou-se por uma análise documental por meio categorial e temática.

A análise categorial trata do desmembramento do discurso em categorias, em que os critérios de escolha e de delimitação orientam-se pela dimensão da investigação dos temas relacionados ao objeto de pesquisa, identificados nos discursos dos participantes pesquisados ${ }^{(12)}$. Tal análise seguiu os quesitos norteadores do roteiro de entrevista ${ }^{(12)}$.

Após a entrevista com os coordenadores de saúde bucal, realizou-se a transcrição e a estruturação das falas em formato de texto. Em seguida, realizou-se o desmembramento, com a categorização e a análise comparativa dos dados, segundo quesitos norteadores obtidos nos diferentes relatos feitos pelos coordenadores de saúde bucal. Essas mensagens foram analisadas de acordo com os objetivos do estudo, com base nas informações obtidas através do instrumento aplicado aos participantes da pesquisa e discutidas à luz da literatura atual, baseada em artigos científicos e portarias ministeriais, e da análise proposta ${ }^{(12)}$. A partir das questões norteadoras, estruturou-se o resultado em cinco categorias: competências de um coordenador de saúde bucal; curso de formação/capacitação para o cargo de gestor; contribuição da formação acadêmica ao cargo de gestor; desafios no processo de trabalho; satisfação por estar no cargo de gestor.

Esta pesquisa obteve aprovação do Comitê de Ética em Pesquisa da Universidade de Fortaleza (UNIFOR), sob o Parecer n. ${ }^{\circ} 3.192 .763$, e todos os entrevistados assinaram o Termo de Consentimento Livre e Esclarecido (TCLE) após explicação detalhada sobre os objetivos da pesquisa, em obediência aos ditames da Resolução n. ${ }^{\circ} 466 / 12$ do Conselho Nacional de Saúde ${ }^{(13)}$, que normatiza os aspectos éticos e bioéticos de pesquisa com seres humanos. Os profissionais entrevistados foram categorizados como $\mathrm{C} 1, \mathrm{C} 2, \mathrm{C} 3, \mathrm{C} 4, \mathrm{C} 5$ e $\mathrm{C} 6$, resguardando-se a identidade dos mesmos.

\section{RESULTADOS E DISCUSSÃO}

\section{Dados de identificação dos participantes}

Participaram seis cirurgiões-dentistas atuantes como coordenadores de saúde bucal, com uma faixa etária de 25 a 64 anos. O período de atuação na gestão variou entre um e vinte anos. Todos apresentaram especializações e mestrado em Saúde da Família, Gestão em Serviços de Saúde ou Gestão Clínica no SUS.

\section{Competências de um coordenador de saúde bucal}

Nesta categoria, pode-se constatar que os entrevistados expressaram o termo "processos de trabalho" no que diz respeito às competências de um coordenador de saúde bucal:

"[...] As principais competências delegadas giram em torno da ajuda nos processos de trabalho dos cirurgiõesdentistas, entre eles, o acolhimento, as atividades de saúde coletiva e a apresentação de propostas e metas a serem alcançadas pelos profissionais." (C1)

“[...] Estão relacionadas ao processo de trabalho dos cirurgiões-dentistas, às cargas horárias, à sistematização dos processos de trabalho, com a implementação dos prontuários eletrônicos, a elaborar escalas entre profissionais, a incentivar as atividades extraclínicas e cursos visando uma melhor capacitação aos profissionais." (C2)

"[...] Gerir os processos de atendimento odontológico das Unidades de Atenção Primária à Saúde [UAPS] acolhimentos, salas de espera, agendamento clínico e visitas domiciliares... Há o encaminhamento paras as especialidades..." (C3)

"[...] Organização de processo de trabalho; manutenção e monitoramento de equipamentos; controle e gerenciamento de insumos; relações humanas; monitoramento das ações gerais, dos indicadores de saúde bucal; além de ajudar nas outras áreas da gestão, quando necessário." (C5)

Um estudo realizado no Rio de Janeiro, Brasil(14), apontou que cirurgiões-dentistas da Estratégia Saúde da Família apresentaram como atividades principais no processo de trabalho as atividades clínicas, ações em creches e escolas, visitas domiciliares e reuniões de equipe. $E$ tais ações eram gerenciadas por um cirurgião-dentista, denominado coordenador de saúde bucal. Esse estudo corrobora as falas dos participantes da presente pesquisa, os quais apontaram competências de um coordenador de saúde bucal.

Notou-se, por meio das falas dos participantes do atual estudo, que o gerenciamento de insumos e equipamentos se destaca dentre as principais atividades realizadas pelos coordenadores de saúde bucal. Assim, todo o abastecimento e manutenção dos componentes do consultório estão sob responsabilidade desses gestores: 
"Solicitação de materiais médico-hospitalares e materiais odontológicos. É realizada uma avaliação dos pedidos de materiais odontológicos mais específicos, de acordo com o número de profissionais $X$ o número de cadeiras $X$ o número da população assistida. Somos responsáveis, também, pelo andamento das ouvidorias. As vistorias dos equipamentos odontológicos (raios $X$, expurgo, cadeira odontológica). Analisa-se, também a escala dos profissionais. Realizamos as visitas técnicas a fim de prevenir e dar suporte às unidades. Implementação do prontuário eletrônico... " (C3)

"Avaliam-se os pedidos com relação à quantidade material $X$ dentista $X$ procedimento realizado. Monitora-se a necessidade de manutenção dos equipamentos de esterilização, por exemplo." (C6)

Também as relações humanas estão diretamente ligadas à coordenação do gestor, e o convívio profissionalprofissional e profissional-paciente deverá ser articulado pelo coordenador, a fim de estabelecer um ambiente de trabalho harmônico:

“[...] Podemos pontuar a gestão de pessoas (cirurgiões-dentistas, ASB/TSB [Auxiliar em Saúde Bucal/Técnico em Saúde Bucal]), a locação das vagas nas diferentes unidades. Promovemos, também, cursos de educação permanente dos profissionais. Há, também, o gerenciamento de insumos odontológicos, mensalmente. Incentivo às ações coletivas interdisciplinares. Além de realizar reuniões de núcleo visando à troca de experiências pelos profissionais das diversas áreas." (C6)

As principais funções delegadas a um coordenador de saúde bucal giram em torno da ajuda nos processos de trabalho dos cirurgiões-dentistas, como a realização de atividades de saúde coletiva (acolhimento e visitas domiciliares) e a exposição de propostas e metas a serem cumpridas pelos profissionais no serviço em que estão atuando(15).

As atividades citadas pelos entrevistados objetivando a melhoria dos serviços ofertados encontram paralelo em um estudo que defende que os pressupostos que regem a reorientação do modelo de atenção em saúde bucal, no Brasil, incluem integralidade, resolutividade e qualificação da atenção básica, indissociavelmente articuladas ao conjunto da rede de serviços ${ }^{(16)}$.

Entre as competências de um coordenador de saúde bucal, constam: coordenar a atenção em saúde bucal básica e especializada e recursos humanos em Odontologia; controlar o fornecimento de material de consumo, instrumental e equipamentos odontológicos; fiscalizar e direcionar a assistência técnica; consolidar procedimentos, por meio dos mapas diários de procedimentos clínicos e extraclínicos; produzir, monitorar e avaliar indicadores de saúde bucal; elaborar relatórios técnicos referentes os resultados dos processos de trabalhos dos cirurgiõesdentistas; contribuir na educação permanente dos cirurgiões-dentistas, entre outras ${ }^{(17)}$.

Como parte essencial da gestão em saúde bucal, a avaliação tem como propósito fundamental dar suporte a todo processo decisório no âmbito do SUS e, por isso, deve subsidiar a identificação de problemas e a reorientação de ações e serviços desenvolvidos, além de avaliar a incorporação de novas práticas sanitárias na rotina de profissionais e mensurar o impacto das ações implementadas pelos serviços e programas sobre o estado de saúde da população( ${ }^{(7,17)}$.

Um estudo realizado no estado de Rio Grande do Norte, Brasil, comparou estratégias de monitoramento e avaliação de indicadores de saúde bucal, apontando que o indicador de cobertura de equipes de saúde bucal do município de Santa Cruz ultrapassou o do Rio Grande do Norte. Pode ser que táticas de execução, monitoramento e avaliação de ações de saúde bucal ainda sejam um problema sério a ser enfrentado pelos gestores. Os autores consideram que tal obstáculo pode ter vários fatores, como falta de mecanismos de acompanhamento, controle e avaliação das ações realizadas pelos serviços odontológicos ${ }^{(18)}$.

Momentos de discussão entre o coordenador de saúde bucal e os cirurgiões-dentistas mostram-se de importante valia para o enriquecimento do processo de trabalho de todos os envolvidos, principalmente nas tomadas de decisão em avaliação em saúde bucal|(5,6).

O atual estudo corrobora outro(19), no qual se afirma que um coordenador local, que pode ser um cirurgiãodentista de formação base, pode ter as seguintes competências: capacidade de estabelecer intermediação, diálogo, negociação e parcerias para resolução de problemas; articulação política em prol da unidade básica de saúde Sistema Municipal de Saúde; e capacidade de liderança, de tomar decisões, de gestão compartilhada, e de atender aos anseios dos cidadãos, especialmente aos usuários do serviço( ${ }^{(19)}$.

Os coordenadores garantem que há pactuação de metas, avaliação dos indicadores de cada profissional e monitoramento deles. Realiza-se o gerenciamento das produções dos profissionais através da alimentação do sistema de prontuário eletrônico. Solicita-se que haja um preenchimento diário do que foi realizado na unidade. A oferta de ações e procedimentos odontológicos é avaliada mensalmente pelos coordenadores. É considerada, 
também, competência de um coordenador de saúde bucal a realização de visitas às unidades, a fim de evidenciar as necessidades operacionais, visando a garantir um atendimento integral aos usuários, elaborando escalas entre profissionais e metas de acordo com as necessidades locais ${ }^{(19)}$.

O coordenador de saúde bucal também deve fazer um monitoramento na Atenção Primária de Saúde, que contemple, minimamente, aspectos como: estimativa de cobertura populacional de equipe de saúde bucal (ESB); percentual de crianças livres de cárie dentária; taxa de incidência de alterações da mucosa oral; percentual de exodontia realizada em relação aos procedimentos clínicos; média de participantes de ação coletiva de escovação dental supervisionada; cobertura de primeira consulta de atendimento odontológico à gestante; razão entre tratamentos concluídos e primeiras consultas odontológicas programáticas, entre outros ${ }^{(17)}$.

E, no âmbito dos Centros de Especialidades Odontológicas (CEO), o coordenador deve: atuar no número de usuários na fila de espera de encaminhamentos registrados no serviço de regulação; atuar no tempo médio de espera entre o encaminhamento e o início do atendimento no CEO; verificar o percentual de tratamentos não concluídos, redimensionar a disponibilidade de horários por especialidade e solicitar mudança no tipo de CEO levando em conta as necessidades da população, entre outras ações ${ }^{(17)}$.

Em alguns municípios brasileiros, coordenadores de saúde bucal não conseguem exercer atividades de gestão quanto ao planejamento, avaliação e monitoramento de ações e serviço, além de não compreenderem o modelo de atenção à saúde bucal preconizado pelo Brasil Sorridente. Em decorrência, resultam práticas pouco efetivas e não condizentes com a necessidade real de saúde bucal da população ${ }^{(20)}$.

Apontam-se, também, grande demanda acumulada e falta de planejamento como principais dificuldades para a incorporação de atividades extraclínicas no âmbito da saúde bucal nas unidades de Saúde da Família, caracterizando um modelo de atenção à saúde bucal clínico ${ }^{(14)}$. Essas condições dificultam o processo de trabalho do coordenador de saúde bucal, afetando diretamente na execução de suas competências.

\section{Curso de formação/capacitação para o cargo de gestor}

Observa-se, nesta categoria, através dos relatos, que não houve a oferta de uma capacitação prévia à posse do cargo. Apenas um coordenador relatou ter feito capacitação. Os outros entrevistados afirmaram possuir experiências anteriores, assim como especializações e mestrados, que contribuíram para a atuação no cargo de gestão, como apontam as falas abaixo:

"Não houve uma capacitação oferecida pelo serviço antes do momento da posse, existindo, apenas, um repasse de informações a respeito do funcionamento das funções delegadas à gestão. Atualmente, alguns cursos são oferecidos pela SMS, porém, nada específico no momento inicial da gestão." (C1)

"Houve uma capacitação prévia à posse do cargo, no intuito de instruir quanto ao funcionamento das unidades de saúde, à conduta do coordenador e às possíveis dúvidas existentes." (C4)

"Não houve nenhuma capacitação prévia. No entanto cursos são oferecidos durante a gestão e capacitações são realizadas quando necessário (por exemplo, capacitação para sistematização). Fui convidado pela experiência que eu adquiri na gestão de Canindé, já fui Secretário de Saúde e secretário executivo." (C5)

Há coordenadores de saúde bucal de municípios brasileiros que não apresentam capacitação ou pós-graduação baseada nas premissas do SUS, além de apresentarem formação acadêmica curativa, favorecendo uma falta de preparo do profissional para atuação mais adequada no cargo $^{(20)}$.

Nota-se que os currículos universitários ainda são voltados para a fragmentação da assistência odontológica, com um caráter curativista e especialista. A maioria dos participantes da presente investigação recebeu uma formação voltada para as ações curativas e técnicas, com pouca ênfase nos fatores socioeconômicos e psicológicos do processo saúde-doença, além de gestão. Apesar de as Diretrizes Curriculares Gerais da formação em saúde, aprovadas pelo Conselho Nacional de Educação, estabelecerem que os currículos devam dar ênfase à promoção da saúde, princípios e diretrizes do SUS e noções básicas de gestão em saúde, a educação superior em saúde no Brasil ainda segue um modelo de prática hospitalocêntrica e especializada, não sendo voltada para a gestão em saúde. Esse fato pode representar mais um desafio para o cirurgião-dentista da ESF e, principalmente, para o coordenador de saúde bucal, que necessita desenvolver ações e serviços gerenciais multidisciplinares e com uma visão holística. Dessa forma, vê-se a necessidade de capacitação e educação permanente para os coordenadores de saúde bucal, de forma a suprir uma possível fragilidade curricular ${ }^{(14)}$.

Os participantes do atual estudo relataram, dentro do seu processo de trabalho, participações em cursos ofertados pela SMS e Instituições de Ensino Superior (IES) referente às capacitações em diversas áreas da Saúde Pública e 
atualizações específicas em Gestão em Saúde Bucal. É de grande valia que sejam ofertadas possibilidades para uma melhor formação dos novos profissionais de saúde e para qualificação e desenvolvimento dos que já atuam no SUS, por meio de propostas que os impulsionem e direcionem a prestação de serviços de forma mais ampla(21).

\section{Contribuição da formação acadêmica ao cargo de gestor}

Em relação a essa categoria, os coordenadores investigados afirmaram que o curso de graduação contribuiu, de forma relevante, para sua atuação como gestores em saúde bucal, informações que foram obtidas a partir da fala dos envolvidos na pesquisa. Os demais participantes afirmam que não possuíam preparação no período de graduação e, sim, em suas vivências fora da faculdade, após formados. De todos os coordenadores entrevistados, apenas um relatou que a matriz curricular de seu curso de graduação era direcionada à saúde coletiva e influenciou, diretamente, na sua atuação como coordenador de saúde bucal. Os demais entrevistados afirmaram que a formação base em Odontologia é fundamental para exercício da função:

"A minha formação acadêmica foi essencial, pois tive a oportunidade de vivenciar na prática o que foi repassado durante as aulas teóricas." (C1)

"Lembro que, na época da faculdade, não houve direcionamento na área de gestão. Meus vinte e quatro anos de vivências, minha gestão de consultório e gerenciamento na policlínica me possibilitou uma experiência para assumir o cargo." (C2)

"A minha formação não contribuiu para o âmbito de gestão. O interesse foi sendo despertado aos poucos, ao longo do meu trabalho no município de Canindé." (C5)

"A minha formação acadêmica não influenciou quase nada. O que realmente contribuiu foram as minhas vivências na coordenação no município de Maranguape, associada à vontade de atuar como gestora." (C6)

Observa-se que, no campo da Odontologia, a reformulação dos cursos de graduação é indispensável, tornandoos cursos com caráter interprofissional e com trabalho colaborativo, para que sejam formados profissionais aptos a exercerem as práticas que compõem o SUS, ressaltando, também, a importância da constante capacitação dos já formados que atuam no serviço ${ }^{(22)}$.

Com esse intuito, o Ministério da Saúde (MS) compõe estratégias de diálogo com o Ministério da Educação (MEC), na tentativa de viabilizar o cumprimento das metas constitucionais. Tanto nos postos de trabalho quanto nos cursos técnicos e universidades foram estabelecidas medidas para reorganizar a formação, o desenvolvimento e a qualificação dos profissionais da área da saúde, a fim de combater o modelo biomédico hegemônico ${ }^{(23)}$.

Apesar dos avanços em Instituições de Ensino Superior brasileiras, a formação dos profissionais de saúde ainda é fragmentada no que se refere à integralidade no atendimento e à gestão em saúde. Há uma necessidade de capacitação e educação contínua com o intuito de (re)estruturar o perfil de trabalho de profissionais de saúde para uma qualificação dos serviços prestados ao SUS, incluindo a gestão em saúde bucal(24).

As mudanças realizadas nas matrizes curriculares dos cursos da saúde, inserindo disciplinas e módulos de Saúde Coletiva, foram necessárias para as melhorias na formação acadêmica de um cirurgião-dentista. Tal condição é relatada apenas pelo entrevistado $\mathrm{C} 1$, que, com menos de dois anos de graduado, em comparação aos demais coordenadores, durante o período da faculdade cursou disciplinas de Saúde Coletiva com o objetivo de desenvolver habilidades de gestão para a sua atuação no SUS.

Além dos obstáculos referentes à deficiência na formação e capacitação dos profissionais atuantes na saúde pública, o financiamento mostra-se, ainda, como o principal entrave ao funcionamento de uma gestão de saúde bucal satisfatória.

\section{Desafios no processo de trabalho}

Nesta categoria, os coordenadores de saúde bucal afirmaram que os principais obstáculos vivenciados durante a gestão se concentram na quantidade insuficiente de insumos a fim de abranger o maior número de usuários. Outro fator relevante é necessidade da manutenção dos equipamentos, que ficam parados nos consultórios, inviabilizando, assim, o atendimento à população:

"Na função de um coordenador de saúde bucal destacamos alguns entraves, em que a resolução de problemas semelhantes é bem recorrente, entre eles podemos enfatizar a falta de insumos hospitalares de uso diário, materiais odontológicos e equipamentos, além de lidar com as possiveis reclamações provenientes dos profissionais e, até mesmo, dos pacientes." (C1) 
"Um dos principais entraves concentra-se na gestão de recursos humanos. Entre eles estão os profissionais e os auxiliares, o que requer a compreensão de ambos. A falta de regularidade no abastecimento dos materiais e problemas estruturais das UAPS com parte elétrica e queda de corrente." (C3)

"[...] Temos uma pequena cobertura da área, apenas 26\%. Há uma grande população para uma reduzida quantidade de equipes de saúde da familia, além da ineficiência de cobertura da média e alta complexidade em saúde bucal." (C5)

"As principais dificuldades encontradas giram em torno do financiamento da saúde bucal, aproximadamente, cem ESB sem cadastro, em que a verba liberada não condiz com a real necessidade das UAPS do município." (C6)

Obstáculos ainda precisam ser superados, tanto no que diz respeito à fragilidade da qualidade do serviço quanto à escassez dos recursos financeiros disponibilizados pelos governantes, indo ao encontro de um estudo que relata que a maioria dos coordenadores municipais de saúde bucal não sabe indicar o percentual do orçamento municipal da saúde destinado à saúde bucal ${ }^{(5)}$. Relevante também é a falta de perfis adequados dos profissionais envolvidos nas questões de gestão em saúde bucal, o que impossibilita a realização e manutenção dos programas de saúde bucal ofertados às populações ${ }^{(25)}$.

A dificuldade de encaminhamento de pacientes para a média e a alta complexidade é presente. O referenciamento e contrarreferenciamento para as unidades especializadas ocorrem por meio da central de regulação, com o foco na disponibilização de vagas, o que caracteriza uma demanda programada. Mas quando se refere à alta complexidade, não há uma organização via sistema, obedecendo, assim, uma demanda espontânea ou encaminhada informalmente, por meio de telefone e/ou e-mai( ${ }^{(26)}$. Acredita-se que a ausência no planejamento das ações e serviços pode influenciar no processo de referenciamento.

Em quatro municípios do estado de Pernambuco, a falta de uma rede de referência e contrarreferência oficializada para a saúde bucal é presente, o que compromete a qualidade da assistência prestada e não garante à população uma atenção nos demais níveis de complexidade na rede de atenção à saúde bucal ${ }^{(16,20)}$.

Outro obstáculo relatado é o subfinanciamento da saúde bucal, pois existem várias condições de não conformidades nas UAPS, como unidades e ESB sem cadastros, de modo que a verba liberada não condiz com a real necessidade da população. Não há uma parcela fixa e específica do financiamento para a saúde bucal no todo advindo da atenção básica. Tal contexto é motivo de discussões em congressos e até em Conferências Nacionais de Saúde ${ }^{(27)}$. O coordenador de saúde bucal, na maioria das vezes, desconhece se realmente o dinheiro está sendo aplicado na saúde bucal, mesmo o financiamento sendo oriundo do Piso da Atenção Básica (PAB).

\section{Satisfação por estar no cargo de gestão}

De acordo com o relato dos entrevistados, nesta categoria, a maior satisfação em estar frente ao cargo de gestão é, sobretudo, o convívio com profissionais de diversas áreas, contribuindo com o constante aprendizado individual:

"A minha maior satisfação em estar à frente da coordenação é, basicamente, pela oportunidade de conviver com profissionais de diversas áreas, possibilitando-me aprender um pouco mais a cada dia." (C1)

"Minha satisfação em atuar frente à gestão é devido ao aprendizado diário, muito válido pela riqueza de pessoas que se convive. O trabalho torna-se desafiante por uma série de fatores, entre eles posso destacar a implementação do teste biológico, que foi um desafio." (C2)

Os coordenadores entrevistados afirmam que as melhorias no atendimento ao público são de grande valia no tocante ao sentimento de êxito pessoal e profissional quando observam o fortalecimento e qualificação do serviço, como apontam os relatos abaixo:

"Uma das maiores satisfações em atuar como gestora são os desafios enfrentados, pois é um trabalho cansativo, que exige muito de você." (C3)

"A minha satisfação frente a esse cargo é poder contribuir com a gestão para a melhoria dos atendimentos, tornando fundamental a atuação da Odontologia nas unidades básicas de saúde. Ver o serviço acontecendo, o profissional cirurgião-dentista atendendo e a população, sendo assim, assistida." (C4)

"É complicado, mas eu gosto. Temos que gostar realmente do que fazemos, há muitas dificuldades. Traz um bem-estar pessoal saber que você pode estar ajudando pessoas. As maiores dificuldades não são com relação aos processos de trabalho, mas, sim, com as relações humanas." (C5)

"Minha maior satisfação frente à coordenação de saúde bucal da regional é pessoal, com o intuito maior de 
ampliar os serviços odontológicos de qualidade, reverberando, assim, as nossas ações. Sinto-me plenamente realizada na minha função, porém não é um trabalho fácil." (C6)

A satisfação no trabalho está susceptível às situações e experiências vivenciadas dentro e fora do ambiente de trabalho, podendo influenciar, também, na rotina social e afetar a qualidade de vida. De modo que, uma vez que o profissional está satisfeito com o seu trabalho, possivelmente haverá estímulos na busca por melhorias nos serviços prestados ao sistema de saúde ${ }^{(28)}$. A existência de certa insatisfação pela falta de insumos, principalmente médicohospitalar, é presente nos relatos. Tal condição afeta diretamente a oferta de serviços odontológicos à população e faz com que os coordenadores de saúde bucal sejam alvo de assédios de profissionais e usuários do SUS, o que exige uma postura de mediador de conflitos.

De acordo com as entrevistas dos participantes, observa-se que, mesmo com as dificuldades que surgem durante o período do cargo de gestão, os coordenadores afirmam gostar das atividades executadas e referem bemestar pessoal ao saberem que estão ajudando outros indivíduos. No entanto afirmam que as maiores dificuldades estão relacionadas com os processos de trabalho e com as relações interpessoais. Constatou-se, também, que a maior satisfação frente ao cargo de gestão é a contribuição com a melhoria dos atendimentos, ampliação dos serviços odontológicos com qualidade e a possibilidade de conviver com profissionais de diversas áreas.

Esses resultados corroboram outros estudos ${ }^{(29,30)}$, em que os cirurgiões-dentistas entrevistados consideraram significativos os trabalhos desenvolvidos no sistema público de saúde, principalmente o acolhimento, por beneficiar os usuários, sendo considerado êxito pessoal e realização profissional no trabalho.

Conhecer as competências e cargo dos gestores em saúde bucal são desafios para pesquisadores, contudo pode provocar mudanças na promoção da saúde, além de apoiar técnicos e gestores na formulação de novas práticas balizadas e com potencial para provocar mudanças no perfil de saúde bucal da população brasileira ${ }^{(31)}$.

O presente estudo apresenta limitações, tendo em vista ter sido realizado com apenas seis gestores coordenadores de saúde bucal, que podem não representar o número de gestores de saúde bucal do estado do Ceará, entretanto não era objetivo do estudo analisar todo o Estado. Os resultados aqui apresentados podem contribuir para o planejamento e implementação de políticas de formação e melhor inserção do coordenador de saúde bucal nos serviços odontológicos públicos, principalmente na ESF.

\section{CONSIDERAÇÕES FINAIS}

Os coordenadores de saúde bucal investigados consideram o cargo importante para a gestão de saúde pública, apesar de encontrarem obstáculos no cotidiano de trabalho, como quantidade insuficiente de insumos e subfinanciamento do setor. Relações humanas, gerenciamento de insumos e equipamentos e a gestão dos processos de atendimento odontológico foram apresentados como principais competências e são essenciais para uma boa qualidade do serviço público de saúde bucal.

É substancial a necessidade de se qualificar os cirurgiões-dentistas para assumir o cargo de gestor em saúde bucal no SUS, para que possam potencializar a atenção primária em saúde como porta de entrada no serviço, promovendo ações de promoção, prevenção e recuperação da saúde do usuário.

\section{CONFLITOS DE INTERESSE}

Os autores deste trabalho não possuem conflitos de interesses.

\section{CONTRIBUIÇÕES}

Davi Oliveira Bizerril contribuiu com a elaboração e delineamento do estudo; a aquisição, análise e interpretação de dados; a redação e revisão do manuscrito. Francisco Cristóvão Mota Lima Júnior contribuiu com a elaboração e delineamento do estudo, e a redação do manuscrito. Margarida Maria Saraiva e Dulce Maria de Lucena Aguiar contribuíram com a aquisição, análise e interpretação de dados, e a revisão do manuscrito.

\section{REFERÊNCIAS}

1. Oliveira L, Passador C. Considerations on the Brazilian national health system (SUS) performance index. Sante Publique (Bucur). 2014;26(6):829-36. 
2. Soares SS, Lima LD, Castro ALB. The role of primary health care in urgency assistance: a look at the policies. J Manag Prim Health Care. 2014;5(2):170-1.

3. Aquilante AG, Aciole GG. Building a "Smiling Brazil"? Implementation of the Brazilian National Oral Health Policy in a health region in the State of São Paulo. Cad Saúde Pública [Internet]. 2015 [acesso em 2019 Jul 02];31(1):82-96. Disponível em: http://dx.doi.org/10.1590/0102-311X00193313

4. Ministério da Saúde (BR). Secretaria de Atenção à Saúde. Departamento de Atenção Básica. Política Nacional de Saúde Bucal. Brasilia: Ministério da Saúde; 2004.

5. Alves JG, Romero VHD, Boeira PO, Bighetti TI, Castilhos ED. The professional satisfaction of primary health care dentists in a city in southern Brazil with municipal oral health guidelines. RFO UPF. 2018;23(2):139-43.

6. Baldani MH, Ribeiro AE, Gonçalves JRSN, Ditterich RG. Oral health work process in Primary Health Care: intermunicipal inequalities highlighted by the PMAQ-AB. Saúde Debate [Internet]. 2018 [acesso em 2019 Jul 16];42(spe1):145-62.

7. Pires DA, Colussi CF, Calvo MCM. Assessment of municipal management of oral health in primary care: data collection instrument accuracy. Ciênc Saúde Colet [Internet]. 2014 [acesso em 2019 Jul 16];19(11):4525-34. Disponível em: http://www.scielo.br/scielo.php?script=sci_arttext\&pid=S1413-81232014001104525

8. Moura ELS, Oliveira EEG, Safh F, Nascimento LS, Brandão GAM. Práticas de odontologia em Saúde Coletiva na Estratégia Saúde da Família. Rev ABENO. 2015;15(3):52-9.

9. Minayo MCS, Diniz D, Gomes R. The qualitative research article under scrutiny. Ciênc Saúde Colet [Internet]. 2016 [acesso em 2019 Jun 30];21(8). Disponível em: https://doi.org/10.1590/1413-81232015218.15592016

10. Saraiva MM. Análise do acesso da pessoa idosa aos serviços de saúde na Atenção Primária [dissertação]. Fortaleza: Universidade de Fortaleza; 2017.

11. Batista EC, Matos LAL, Nascimento AB. A Entrevista como técnica de investigação na pesquisa qualitativa. Rev Interdisciplin Cient Apl. 2017;11(3):23-38.

12. Bardin L. Análise de conteúdo. São Paulo: Edições 70; 2011.

13. Conselho Nacional de Saúde (BR). Resolução $n^{\circ} 466$, de 12 de dezembro de 2012. Institui diretrizes e normas regulamentadoras de pesquisas envolvendo seres humanos. Brasília: Ministério da Saúde; 2012.

14. Moraes LB, Kligerman DC, Cohen SC. Analysis on the socio-demographic profile and dental surgeon's work process in the Family Health Program, in three cities of the mountain region of the state of Rio de Janeiro. Physis [Internet]. 2015 [acesso em 2019 Jul 02];25(1):171-86. Disponível em: http://www.scielo.br/scielo. php?pid=S0103-73312015000100171\&script=sci_abstract

15. Brasil. Ministério da Saúde. Portaria n $n^{\circ}$ 3.012, de 26 de dezembro de 2012. Redefine a composição das equipes de Saúde Bucal da Estratégia Saúde da Família constante na Política Nacional de Atenção Básica (PNAB). Diário Oficial da União; Brasília, 27 de dezembro de 2012.

16. Godoi H, Andrade SR, Mello ALSF. The regionalized healthcare network in Santa Catarina State, Brazil, from 2011 to 2015: governance system and oral healthcare. Cad Saúde Pública [Internet]. 2017 [acesso em 2019 Jul 16];33(9):e00133516. Disponível em: http://www.scielo.br/scielo.php?script=sci_arttext\&pid=S0102$311 \times 2017000905009$

17. Ministério da Saúde (BR). Secretaria de Atenção à Saúde. Departamento de Atenção Básica. A saúde bucal no Sistema Único de Saúde [Internet]. Brasília: Ministério da Saúde; 2018 [acesso em 2019 Jun 30]. Disponível em: http://bvsms.saude.gov.br/bvs/publicacoes/saude_bucal_sistema_unico_saude.pdf

18. Araújo IDT, Machado FCA. Temporal evolution of oral health indicators in municipalities of Rio Grande do Norte. Rev Ciênc Plur. 2018;4(2):73-86.

19. Fischer SD, Horn FB, Schommer PC, Santos JGM. Competências para o cargo de coordenador de Unidade Básica de Saúde. TAC. 2014;4(2):117-31.

20. Santos NML, Hugo FN. Training in Family Health and its association with work processes of Primary Care Dentistry Teams. Cienc Saúde Colet. 2018;23(12):4319-29. 
21. Maciel JAC, Vasconcelos MIO, Castro-Silva II, Eloia SMC, Farias MR. Permanent education in the Healthcare area for Dentists of the Family Health Strategy: an integrate review. Rev APS. 2017;20(3):414-22.

22. Holanda ICLC, França CL, Albuquerque SHC, Fernandes VG, Nuto SAS. Desenvolvimento de habilidades na formação de estudantes de Odontologia: a contribuição da Terapia Ocupacional e da Psicologia. Rev ABENO. 2019;19(x):40-8.

23. Vendruscolo C. Integração ensino-serviço: movimentos das instâncias de gestão nos processos de reorientação da formação profissional na saúde [tese]. Santa Catarina: Universidade Federal de Santa Catarina; 2014.

24. Penedo RM, Gonçalo CS, Quelus DP. Shared management: perceptions of professionals in the family health context. Interface (Botucatu). 2019;23:e170451.

25. Macedo GL, Lucena EES, Lopes IKR, Batista LTO. Access to dental care of special patients: the perception of primary care dentists. Rev Ciênc Plur. 2018;4(1):67-80.

26. Lucena EHG, Lucena CDR, Goes PSA, Sousa MF. Conditions associated with Activity Planning in Dental Specialty Centers. Univ Odontol. 2019;38(80):1-27.

27. Cayetano MH, Carrer FAC, Gabriel M, Carneira FM, Pucca GA Jr. Brazilian National Oral Health Policy (Smiling Brazil): A rescue of history, learning and future Univ. Odontol. 2019;38(80):1-23.

28. Lima AJ, Zille LP, Soares MG. Look on the satisfaction at work: study in a multinational organization of machining sector in the state of Minas Gerais. Gest Planejamento. 2015;16(3):310-25.

29. Silva LAG, Piva MR, Cabral MCB, Peixoto AC, Santos TS, Santos MJO. Perception of dentists in relation to the Single Health System/Family Health (SUS/PSF) Program in the City of Nossa Senhora do Socorro-SE. Odontol Clín-Cient. 2011;10(4):345-49.

30. Aldosari MA, Tavares MA, Machado ATGM, Abreu MHNG. Factors associated with patients' satisfaction in Brazilian dental primary health care. PLos ONE. 2017;12(11):e0187993.

31. Kusma SZ, Moysés ST, Moysés SJ. Promoção da saúde: perspectivas avaliativas para a saúde bucal na atenção primária em saúde. Cad Saúde Pública. 2012;28(SupS9-S19):1-11.

\section{Endereço para correspondência:}

Davi Oliveira Bizerril

Universidade de Fortaleza - UNIFOR

Curso de Odontologia

Av. Washington Soares, 1321/ Bloco O

Bairro: Edson Queiroz

CEP: 60811-905 - Fortaleza - CE - Brasil

E-mail: davibizerril@unifor.br

Como citar: Bizerril DO, Lima FCM Júnior, Saraiva MM, Aguiar DML. Coordenadores de saúde bucal: percepção sobre gestão e competências no sistema único de saúde. Rev Bras Promoç Saúde. 2019;32:9273. 